\title{
An efficient conversion of carboxylic acids into Weinreb amides
}

\author{
Alan R. Katritzky,* Hongfang Yang, Suoming Zhang, and Mingyi Wang \\ Department of Chemistry, Center for Heterocyclic Compounds, University of Florida, \\ Gainesville, Florida 32611-7200, USA \\ E-mail: katritzky@chem.ufl.edu
}

\section{Dedicated to our good friend Mimmo Spinelli}

(received 11 Sep 02; accepted 15 Nov 02; published on the web 23 Nov 02)

\begin{abstract}
Efficient conversions of carboxylic acids into Weinreb amides were achieved by treatment of $N$ acylbenzotriazoles 2a-i with $\mathrm{N}, \mathrm{O}$-dimethylhydroxylamine hydrochloride under mild conditions. No racemization was found when optically active acids were employed.
\end{abstract}

Keywords: Weinreb amides, $N$-acylbenzotriazoles

\section{Introduction}

Recently, increasing attention has been paid to $N$-methoxy- $N$-methylamides (Weinreb amides) owing to their versatile reactivity with nucleophiles and selective reduction to aldehydes. ${ }^{1}$ Weinreb amides derived from amino acids have found extensive use in the preparation of $\alpha$ amino aldehydes ${ }^{2}$ and $\alpha$-amino ketones. ${ }^{3}$

Most direct conversions of carboxylic acids into the corresponding Weinreb amides have utilized peptide-coupling reagents such as benzotriazol-1-yl- $N$-oxy-tris(dimethylamino)phosphonium hexafluorophosphate (BOP) ${ }^{4} N, N$ '-dicyclohexylcarbodiimide (DCC) ${ }^{5}$ or propylphosphonic anhydride/ $N$-ethylmorpholine. ${ }^{6}$ Other reagents such as carbon tetrabromide/triphenylphosphine, ${ }^{7}$ 2-chloro-1-methylpyridinium iodide (CMPI) and/or 2bromide-1-methylpyridinium iodide (BMPI), ${ }^{8}$ and [bis-(2-methoxyethyl)-aminosulfur trifluoride (deoxo-fluor reagent) ${ }^{9}$ have also been used as coupling reagents in the preparation of $N$ methoxy- $N$-methylamides and involve in situ formation of the acyl halide. More recently, 2mercaptopyridone-1-oxide-based thiouronium salts have been reported as effective coupling reagents for the synthesis of Weinreb amides from carboxylic acids. ${ }^{10}$

Acylbenzotriazoles are neutral acylating reagents. ${ }^{11}$ Recently, our group has demonstrated advantageous methods for the preparation of acylbenzotriazoles from carboxylate anions and used the acylbenzotriazoles for the preparation of primary, secondary and tertiary amides, ${ }^{12}$ and 
cinnamoyl hydrazides. ${ }^{13} \mathrm{~N}$-Formyl-, ${ }^{14 \mathrm{a}}$ or $N$-trifluoroacetyl-benzotriazole, ${ }^{14 \mathrm{~b}}$ and $1,1^{\prime}-(1,2-$ dioxoethane-1,2-diyl)bis-1H-bis-benzotriazole ${ }^{14 c}$ have been used advantageously for acylation. Very recently we have demonstrated that $\alpha$-(Boc-amino) acids can be converted into stable chiral $\alpha$-aminoacylating agents. ${ }^{14 d}$ We now report an efficient procedure for direct conversion of carboxylic acids into the corresponding Weinreb amides, with no racemization when optically active acids are employed.

\section{Results and Discussion}

The acylbenzotriazoles $2 \mathbf{2}-\mathbf{i}$ were readily prepared in $65-91 \%$ yields from the carboxylic acids 1a-i by reaction with BtMs by a previously reported procedure ${ }^{14}$. Reactions of the $\mathrm{N}$ acylbenzotriazoles $\mathbf{2 a}-\mathbf{i}$ with $\mathrm{N}, \mathrm{O}$-dimethylhydroxylamine hydrochloride in THF in the presence of a base at reflux afforded the corresponding Weinreb amides 3a-i in 73-97\% yields (Scheme 1, Table 1). The benzotriazole by-product formed in the reaction can easily be removed and recovered by washing with saturated $\mathrm{Na}_{2} \mathrm{CO}_{3}$. The product isolation can be carried out by column chromatography or recrystallization.

This methodology is applicable to a variety of carboxylic acids with sensitive functional groups. For example, $N$-protected amino acid amides can be prepared readily from the corresponding amino acids (entries $\mathbf{h}-\mathbf{j}$ ). These reactions proceed without detectable racemization of the chiral center, as evidenced by comparison with literature optical rotation values. ${ }^{9}$

In conclusion, we have developed a practical and convenient method for the synthesis of various Weinreb amides from the corresponding carboxylic acids without detectable racemization when chiral substrates are employed using benzotriazole methodology. The simplicity, easy operation, mild reaction conditions, and low cost are advantageous. The use of the previously mentioned peptide coupling reagents also gives high yields with retention of chirality in optically active substrates; however, difficult-to-remove by-products, toxic coproducts (e.g., hexamethylphosphoramide for the BOP reagent), or the high cost of scale-up, can be disadvantageous.

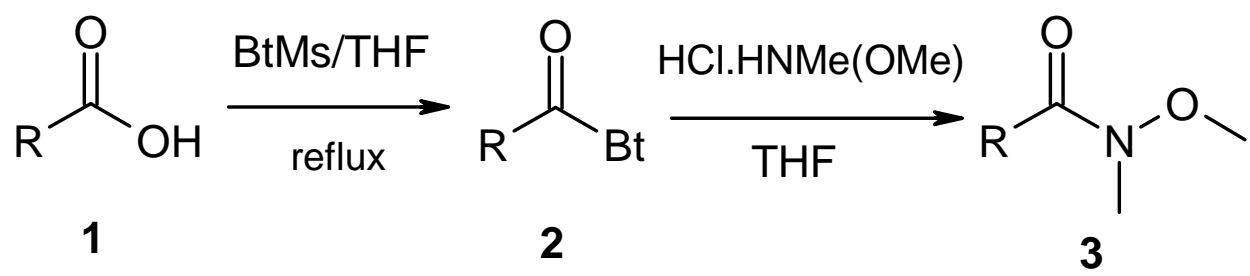

\section{Scheme 1}


Table 1. Yields of the $N$-acylbenzotriazoles 2 and Weinreb amides 3

\begin{tabular}{|c|c|c|c|c|c|c|c|}
\hline \multirow{2}{*}{ Entry } & \multirow{2}{*}{ Acid 1} & \multicolumn{3}{|c|}{2 (Yield, \%) } & \multicolumn{3}{|c|}{3 (Yield, \%) } \\
\hline & & PW & LW & Ref. & PW & LW & Ref. \\
\hline $\mathrm{a}$ & & 89 & 89 & 12 & 78 & 80 & 8 \\
\hline $\mathrm{b}$ & & 91 & 91 & 12 & 74 & 91 & 17 \\
\hline $\mathrm{c}$ & & 89 & 89 & 12 & 73 & 95 & 16 \\
\hline $\mathrm{d}$ & & 96 & 89 & 15 & 82 & 76 & 9 \\
\hline $\mathrm{e}$ & & 93 & 84 & 12 & 77 & l & / \\
\hline $\mathrm{f}$ & & 94 & 90 & 13 & 89 & 76 & 8 \\
\hline $\mathrm{g}$ & & 98 & 84 & 12 & 83 & 86 & 9 \\
\hline $\mathrm{h}$ & & 65 & l & l & 80 & 75 & 8 \\
\hline $\mathrm{i}$ & & 83 & / & / & 71 & 99 & 18 \\
\hline $\mathrm{j}$ & & 81 & / & / & 75 & 89 & 9 \\
\hline
\end{tabular}

* PW, Present Work; LW, Literature Work 


\section{Experimental Section}

General Procedures. Melting points were determined on a MEL-TEMP capillary melting point apparatus equipped with a Fluke 51 digital thermometer. NMR spectra were recorded in $\mathrm{CDCl}_{3}$ with tetramethylsilane as the internal standard for ${ }^{1} \mathrm{H}(300 \mathrm{MHz})$ and the solvent for ${ }^{13} \mathrm{C}(75$ $\mathrm{MHz}$ ) NMR. THF was distilled from sodium/benzophenone under nitrogen immediately prior to use. All reactions with air-sensitive compounds were carried out under argon atmosphere. Column chromatography was conducted on silica gel (230-400 mesh). For the preparation and characterization of $N$-acylbenzotriazoles $\mathbf{2 a}-\mathbf{g}$ see the literature. ${ }^{12,13,15}$

\section{Typical procedure for the preparation of $\mathbf{N}$-acylbenzotriazoles $\mathbf{2 a}-\mathbf{i}$}

To a solution of the acid $1(10 \mathrm{mmol})$ in THF $(10 \mathrm{~mL})$, BtMs $(11 \mathrm{mmol})$ and $\mathrm{Et}_{3} \mathrm{~N}(11 \mathrm{mmol})$ were added at room temperature. The reaction mixture was refluxed 6-12 h. The solvent was removed in vacuo to dryness. The residue was dissolved in ethyl acetate and washed sequentially with satd. citric acid, satd. $\mathrm{Na}_{2} \mathrm{CO}_{3}$ and $\mathrm{H}_{2} \mathrm{O}$, and dried over $\mathrm{MgSO}_{4}$. Concentration under reduced pressure gave the desired product, which was recrystallized from hexane-ethyl acetate.

L-tert-Butyl- $\boldsymbol{N}$-[2-(benzotriazol-1-yl)-1-methyl-2-oxoethyl]carbamate (2h). Needles from hexane-ethyl acetate (61\%), m.p. $68-69^{\circ} \mathrm{C},[\alpha]^{\mathrm{D}}{ }_{25}=-17.7^{\circ}\left(\mathrm{CHCl}_{3}\right) ;{ }^{1} \mathrm{H}$ NMR $\delta 8.27(\mathrm{~d}, J=8.1$ $\mathrm{Hz}, 1 \mathrm{H}), 8.13(\mathrm{~d}, J=8.2 \mathrm{~Hz}, 1 \mathrm{H}), 7.67$ (dd, $J=7.5,7.6 \mathrm{~Hz}, 1 \mathrm{H}), 7.52(\mathrm{dd}, J=7.5,7.6 \mathrm{~Hz}, 1 \mathrm{H})$, $5.74(\mathrm{~m}, 1 \mathrm{H}), 5.36(\mathrm{brs}, 1 \mathrm{H}), 1.66(\mathrm{~d}, J=7.2 \mathrm{~Hz}, 3 \mathrm{H}), 1.45(\mathrm{~s}, 9 \mathrm{H}) ;{ }^{13} \mathrm{C}$ NMR $\delta 172.7,155.1$, 146.0, 131.2, 130.6, 126.4, 120.3, 114.4, 80.3, 50.1, 28.2, 18.8. Anal. Calcd for $\mathrm{C}_{14} \mathrm{H}_{18} \mathrm{~N}_{4} \mathrm{O}_{3}$ : C, 57.92; H, 6.25; N, 19.30. Found: C, 58.06; H, 6.44; N, 19.30.

L-tert-Butyl- $\boldsymbol{N}$-[1-(1H-benzotriazol-1-ylcarbonyl)-2-methylpropyl]carbamate (2i). Prisms from hexane-ethyl acetate (83\%), m.p. $120-121{ }^{\circ} \mathrm{C},[\alpha]^{\mathrm{D}}{ }_{25}=-47.5^{\circ}\left(\mathrm{CH}_{3} \mathrm{OH}\right) ;{ }^{1} \mathrm{H}$ NMR $\delta 8.28$ $(\mathrm{d}, J=8.1 \mathrm{~Hz}, 1 \mathrm{H}), 8.14(\mathrm{~d}, J=8.2 \mathrm{~Hz}, 1 \mathrm{H}), 7.68(\mathrm{dd}, J=7.4,7.3 \mathrm{~Hz}, 1 \mathrm{H}), 7.54$ (dd, $J=7.4,7.5$ $\mathrm{Hz}, 1 \mathrm{H}), 5.70-5.66(\mathrm{~m}, 1 \mathrm{H}), 5.33(\mathrm{~d}, J=8.4 \mathrm{~Hz}, 1 \mathrm{H}), 2.47-2.17(\mathrm{~m}, 1 \mathrm{H}), 1.46(\mathrm{~s}, 9 \mathrm{H}), 1.11(\mathrm{~d}, J$ $=6.7 \mathrm{~Hz}, 3 \mathrm{H}), 0.97(\mathrm{~d}, J=6.7 \mathrm{~Hz}, 3 \mathrm{H}) ;{ }^{13} \mathrm{C} \mathrm{NMR} \delta 171.9,155.6,145.9,130.9,130.5,126.3$, 120.2, 114.3, 80.1, 58.9, 31.4, 28.2, 19.6, 16.9. Anal. Calcd for $\mathrm{C}_{16} \mathrm{H}_{22} \mathrm{~N}_{4} \mathrm{O}_{3}$ : C, 60.36; H, 6.96; N, 17.60. Found: C, 60.56; H, 7.13; N, 17.72.

L-tert-Butyl- $\mathbf{N}$-[2-(1H-benzotriazol-1-yl)-1-benzyl-2-oxoethyl]carbamate (2j). White solid from hexane-ethyl acetate (81\%), m.p. $143.5-144.5{ }^{\circ} \mathrm{C} ;{ }^{1} \mathrm{H}$ NMR $\delta 8.26(\mathrm{~d}, J=7.8 \mathrm{~Hz}, 1 \mathrm{H})$, $8.16(\mathrm{~d}, J=8.1 \mathrm{~Hz}, 1 \mathrm{H}), 7.68(\mathrm{dd}, J=6.9,7.8 \mathrm{~Hz}, 1 \mathrm{H}), 7.55(\mathrm{dd}, J=7.5,7.8 \mathrm{~Hz}, 1 \mathrm{H}), 7.28-7.18$ $(\mathrm{m}, 5 \mathrm{H}), 6.03$ (brs, 1H), 5.28 (brs, 1H), 3.48-3.30 (m, 1H), 3.22-3.09 (m, 1H), $1.41(\mathrm{~s}, 9 \mathrm{H}) .{ }^{13} \mathrm{C}$ NMR $\delta 171.5,155.3,146.2,135.5,131.3,131.2,130.9,129.5,128.9,127.5,126.7,120.6,114.6$, 80.6, 55.5, 39.1, 28.4. Anal. Calcd for $\mathrm{C}_{20} \mathrm{H}_{22} \mathrm{~N}_{4} \mathrm{O}_{3}$ : C, 65.56; H, 6.05; N, 15.29. Found: C, 65.68; $\mathrm{H}, 6.38 ; \mathrm{N}, 14.90$.

\section{Typical procedure for the preparation of Weinreb amides 3a-i}

To a solution of $N$-acylbenzotriazole $2(2 \mathrm{mmol})$ in dry THF $(10 \mathrm{~mL})$, a solution of $N, O-$ dimethylhydroxylamine in THF (prepared from $2.2 \mathrm{mmol}$ of $\mathrm{N}$,O-dimethylhydroxylamine 
hydrochloride, and $2.2 \mathrm{mmol}$ of triethylamine in $5 \mathrm{~mL}$ of dry THF was added at $20{ }^{\circ} \mathrm{C}$ over 5 $\min$. The reaction mixture was stirred at r.t. for $24 \mathrm{~h}$. The solvent was removed in vacuo and the residue was dissolved in EtOAc. The organic layer was washed sequentially with saturated citric acid, satd. $\mathrm{Na}_{2} \mathrm{CO}_{3}, \mathrm{H}_{2} \mathrm{O}$, and dried over $\mathrm{MgSO}_{4}$. Evaporation in vacuo to dryness gave the desired products, which were recrystallized from the appropriate solvent.

$N$-Methoxy- $N$-methylbenzamide (3a). Colorless oil ${ }^{8}$ (78\%); $1 \mathrm{H}$ NMR $\delta 7.67$ (d, $J=6.6 \mathrm{~Hz}$, $2 \mathrm{H}), 7.41(\mathrm{~m}, 3 \mathrm{H}), 3.56(\mathrm{~s}, 1 \mathrm{H}), 3.37(\mathrm{~s}, 1 \mathrm{H}),{ }^{13} \mathrm{C}$ NMR $\delta 170.2,134.2,130.8,128.3,128.2,61.2$, 34.0 .

$N$-Methoxy-4, $N$-dimethylbenzamide (3b). Colorless oil ${ }^{9}$ (74\%); ${ }^{1} \mathrm{H}$ NMR $\delta 7.60$ (d, $J=7.8$ $\mathrm{Hz}, 2 \mathrm{H}), 7.21$ (d, $J=7.8 \mathrm{~Hz}, 2 \mathrm{H}$ ), 3.56 (s, $3 \mathrm{H}), 3.35$ (s, $3 \mathrm{H}), 2.39$ (s. $3 \mathrm{H}) ;{ }^{13} \mathrm{C}$ NMR $\delta 170.2$, 141.1, 131.3, 128.9, 128.5, 61.2, 34.1, 21.7.

$N$-Methoxy- $N$-methyl-2,2-diphenyl-acetamide (3c). White crystals (73\%), m.p. $106-107{ }^{\circ} \mathrm{C}$ (lit. $\left.{ }^{16} 108-108.5 .5{ }^{\circ} \mathrm{C}\right) ;{ }^{1} \mathrm{H}$ NMR $\delta 7.32-7.20(\mathrm{~m}, 10 \mathrm{H}), 5.54(\mathrm{~s}, 1 \mathrm{H}), 3.49(\mathrm{~s}, 3 \mathrm{H}), 3.23(\mathrm{~s}, 3 \mathrm{H})$; ${ }^{13} \mathrm{C}$ NMR $\delta 173.1,139.4,128.9,128.4,126.9,61.4,52.8,32.3$.

$\boldsymbol{N}$-Methoxy- $\boldsymbol{N}$-methyl-2-thiophenecarboxamide (3d). Colorless oil ${ }^{9}(82 \%) ;{ }^{1} \mathrm{H}$ NMR $\delta 7.97$ $(\mathrm{dd}, J=1.1,3.8 \mathrm{~Hz}, 1 \mathrm{H}), 7.56(\mathrm{dd}, J=1.1,5.0 \mathrm{~Hz}, 1 \mathrm{H}), 7.11(\mathrm{dd}, J=4.8,4.0 \mathrm{~Hz}, 1 \mathrm{H}), 3.78(\mathrm{~s}$, $3 \mathrm{H}), 3.38$ (s, $3 \mathrm{H}) ;{ }^{13} \mathrm{C}$ NMR $\delta 162.4,134.6,133.4,132.4,127.0,61.7,33.2$.

$N$-Methoxy- $N$-methylisonicotinamide (3e). Colorless oil (77\%); ${ }^{1} \mathrm{H}$ NMR $\delta 8.72(\mathrm{~d}, J=6 \mathrm{~Hz}$, $2 \mathrm{H}), 7.55(\mathrm{~d}, J=6 \mathrm{~Hz}, 2 \mathrm{H}), 3.55(\mathrm{~s}, 3 \mathrm{H}), 3.37(\mathrm{~s}, 3 \mathrm{H}) ;{ }^{13} \mathrm{C} \mathrm{NMR} \delta 173.6,167.1,149.4,141.6$, 125.2, 121.8, 61.1, 32.8.

(E)- $\boldsymbol{N}$-Methoxy- $\boldsymbol{N}$-methyl-3-phenyl-2-propenamide (3f). Colorless oil ${ }^{7}$ (89\%); ${ }^{1} \mathrm{H}$ NMR $\delta$ $7.75(\mathrm{~d}, J=15.6 \mathrm{~Hz}, 1 \mathrm{H}), 7.58$ (d, $J=3.9 \mathrm{~Hz}, 1 \mathrm{H}), 7.56$ (d, $J=1.8 \mathrm{~Hz}, 1 \mathrm{H}), 7.39$ (m, 3H), 7.05 $(\mathrm{d}, J=15.9 \mathrm{~Hz}, 1 \mathrm{H}), 3.77(\mathrm{~s}, 1 \mathrm{H}), 3.32(\mathrm{~s}, 1 \mathrm{H}) ;{ }^{13} \mathrm{C}$ NMR $\delta 167.0,143.5,135.1,129.8,128.8$, 128.0, 115.7, 61.9, 32.5.

$\mathrm{N}$-Methoxy- $\mathrm{N}$-methyl-3-phenylpropionamide (3g). Colorless oil ${ }^{17}(83 \%) ;{ }^{1} \mathrm{H}$ NMR $\delta$ $7.32-7.17$ (m, 5H), $3.60(\mathrm{~s}, 3 \mathrm{H}), 3.18(\mathrm{~s}, 3 \mathrm{H}), 2.96(\mathrm{t}, J=7.5 \mathrm{~Hz}, 2 \mathrm{H}), 2.74$ (t, $J=7.5 \mathrm{~Hz}, 2 \mathrm{H})$; ${ }^{13} \mathrm{C}$ NMR $\delta 173.5,141.2,128.3,128.3,125.9,61.0,33.6,32.0,30.5$.

tert-Butyl $\mathbf{N}$-((1S)-2-[methoxy(methyl)amino]-1-methyl-2-oxoethyl) carbamate (3h). White crystals (71\%), $\alpha^{\mathrm{D}}{ }_{25}=-27.8$ (c=1, methanol) m.p. ${ }^{9} 145-146{ }^{\circ} \mathrm{C} ;{ }^{1} \mathrm{H}$ NMR $\delta 5.26(\mathrm{~d}, J=7.8 \mathrm{~Hz}$, $1 \mathrm{H}), 4.68(\mathrm{~m}, 1 \mathrm{H}), 3.77(\mathrm{~s}, 3 \mathrm{H}), 3.21(\mathrm{~s}, 3 \mathrm{H}), 1.44(\mathrm{~s}, 9 \mathrm{H}), 1.31(\mathrm{~d}, J=6.9 \mathrm{~Hz}, 3 \mathrm{H}) ;{ }^{13} \mathrm{C} \mathrm{NMR} \delta$ $173.7,155.2,79.5,61.6,46.5,32.1,28.3,18.6$.

tert-Butyl $\boldsymbol{N}$-((1S)-1-[methoxy(methyl)amino]carbonyl-2-methylpropyl)carbamate (3i). Oil ${ }^{18}(71 \%) ; \alpha_{25}{ }_{25}=-13.6\left(\mathrm{c}=2.5\right.$, methanol) ${ }^{1} \mathrm{H}$ NMR $\delta 5.14(\mathrm{~d}, J=8.7 \mathrm{~Hz}, 1 \mathrm{H}), 4.56$ (brs, $\left.1 \mathrm{H}\right), 3.76$ (s, 3H), $3.20(\mathrm{~s}, 3 \mathrm{H}), 1.85(\mathrm{~m}, 1 \mathrm{H}), 1.43(\mathrm{~s}, 9 \mathrm{H}), 0.94(\mathrm{~d}, \mathrm{~J}=6.6 \mathrm{~Hz}, 3 \mathrm{H}), 0.90(\mathrm{~d}, \mathrm{~J}=6.6 \mathrm{~Hz}$, $3 \mathrm{H}) .{ }^{13} \mathrm{C}$ NMR $\delta 172.9,155.8,79.4,61.5,54.9,31.9,31.3,28.3,19.4,17.4$.

tert-Butyl $\mathrm{N}$-\{(1S)-1-benzyl-2-[methoxy(methyl)amino]-2-oxoethyl\}carbamate (3j). Yellow crystals (75\%), m.p. $135-135.5{ }^{\circ} \mathrm{C}^{18} ;{ }^{1} \mathrm{H}$ NMR $\delta 7.30-7.16(\mathrm{~m}, 5 \mathrm{H}), 5.23(\mathrm{~d}, J=7.5 \mathrm{~Hz}, 1 \mathrm{H})$, $4.96(\mathrm{~m}, 1 \mathrm{H}), 3.66$ (s, 3H), 3.17 (s, 3H), 3.06 (dd, $J=6.0,13.5 \mathrm{~Hz}, 1 \mathrm{H}), 2.88$ (dd, $J=7.2,13.2$ $\mathrm{Hz}, 1 \mathrm{H}), 1.38(\mathrm{~s}, 9 \mathrm{H}) ;{ }^{13} \mathrm{C}$ NMR $\delta 172.3,155.2,136.5,129.4,128.3,126.7,125.6,79.6,61.5$, $51.5,38.8,32.1,28.3$. 


\section{References}

1. Nahm, S.; Weinreb, S. M. Tetrahedron Lett. 1981, 22, 3815. (b) Sibi, M. P. Org. Prep. \& Proced. Int. 1993, 25, 15.

2. (a) Jurczak, J.; Golebiowski, A. Chem. Rev. 1989, 89, 149. (b) Fisher, L. E.; Muchowski, J. M. Org. Prep. \& Proced. Int. 1990, 22, 399. (c) Hall, B. J.; Sutherland, J. D. Tetrahedron Lett. 1998, 39, 6539.

3. Hamby, J. M.; Hodges, J. C. Heterocycles 1993, 35, 843.

4. (a) Maugras, I.; Poncet, J.; Jouin, P. Tetrahedron 1990, 46, 2807. (b) Shreder, K.; Zhang, L.; Goodman, M. Tetrahedron Lett. 1998, 39, 221.

5. Braun, M.; Waldmüller, D. Synthesis 1989, 856.

6. (a) Oppolzer, W.; Cunningham, A. F. Tetrahedron Lett. 1986, 27, 5467. (b) Dechantsreiter, M. A.; Burkhart, F.; Kessler, H. Tetrahedron Lett. 1998, 39, 253.

7. Einhorn, J.; Einhorn, C.; Luche, J.-L. Synth. Commun. 1990, 20, 1105.

8. Sibi, M. P.; Stessman, C. C.; Schultz, J. A.; Christensen, J. W.; Lu, J.; Marvin, M. Synth. Commun. 1995, 25, 1255

9. Tunoori, A. R.; White, J. M.; Georg, G. I. Org. Lett. 2000, 2, 4091 and references cited therein.

10. Bailen, M. A.; Chinchilla, R.; Dodsworth, D. J.; Najera, C. Tetrahedron Lett. 2001, 42, 5013.

11. Staab, H. A.; Bauer, H.; Scahneider, K. M. Azolides in Organic Synthesis and Biochemistry; Wiley-VCH: Germany, 1998; pp 129-205.

12. Katritzky, A. R.; He. H.-Y.; Suzuki, K. J. Org. Chem. 2000, 65, 8210.

13. Katritzky, A. R.; Wang, M. Y.; Zhang, S. M. ARKIVOC in press.

14. (a) Katritzky, A. R.; Chang, H.-X.; Yang, B. Synthesis 1995, 503. (b) Katritzky, A. R.; Yang, B.; Semenzin, D. J. Org. Chem. 1997, 62, 726. (c) Katritzky, A. R.; Levell, J. R.; Pleynet, D. P. M. Synthesis 1998, 153. (d) Katritzky, A. R.; Wang, M.; Yang, H.; Zhang, S. M.; Akhmedov, N. G. J. Am. Chem. Soc. submitted.

15. Katritzky, A. R.; Huang, T.; Voronkov, M. V.; Steel, P. J. J. Org. Chem. 2000, 65, 8069.

16. Richter, S. B. US 3177 855, 1961; Chem. Abstr. 1960, 60; 7959h.

17. Williams, J. M.; Jobson, R. B.; Yasuda, N.; Marchesini, G.; Dolling, U.-H.; Grabowski, E. J. J. Tetrahedron Lett. 1995, 36, 5461.

18. Ciapetti, P.; Taddei, M.; Ulivi, P. Tetrahedron Lett. 1994, 35, 3183. 